\title{
Angle-dependent magnetization dynamics with mirror-symmetric excitations in artificial quasicrystalline nanomagnet lattices
}

\author{
V. S. Bhat ${ }^{1,2, *}$ and D. Grundler ${ }^{1,3, \dagger}$ \\ ${ }^{1}$ Laboratory of Nanoscale Magnetic Materials and Magnonics, Institute of Materials (IMX), École Polytechnique Fédérale de Lausanne \\ (EPFL), 1015 Lausanne, Switzerland \\ ${ }^{2}$ Lehrstuhl für Physik funktionaler Schichtsysteme, Physik Department E10, Technische Universität München, 85748 Garching, Germany \\ ${ }^{3}$ Institute of Microengineering (IMT), EPFL, 1015 Lausanne, Switzerland
}

(Received 20 April 2018; revised manuscript received 10 July 2018; published 6 November 2018)

\begin{abstract}
We report angle-dependent spin-wave spectroscopy on aperiodic quasicrystalline magnetic lattices, i.e., Ammann, Penrose P2 and P3 lattices made of large arrays of interconnected $\mathrm{Ni}_{80} \mathrm{Fe}_{20}$ nanobars. Spin-wave spectra obtained in the nearly saturated state contain distinct sets of resonances with characteristic angular dependencies for applied in-plane magnetic fields. Micromagnetic simulations allow us to attribute detected resonances to mode profiles with specific mirror symmetries. Spectra in the reversal regime show systematic emergence and disappearance of spin-wave modes indicating reprogrammable magnonic characteristics.
\end{abstract}

DOI: 10.1103/PhysRevB.98.174408

\section{INTRODUCTION}

Magnetic quasicrystals exhibit aperiodicity and rotational symmetry not observed in periodic crystal. At the same time, they show a complex form of frustration [1,2]. The recently introduced artificial magnetic quasicrystals (AMQs) allow researchers to follow a material by design approach [3] and investigate effects of both aperiodicity and frustration $[4,5]$. Such planar structures offer exploration of magnetic states by direct imaging [6,7] and thereby open the door for optimization of lattices for applications, such as magnonic crystals [8] for data processing with spin waves (SWs) [9]. Reprogrammable magnetic states in such periodically modulated media enable magnonic excitations tailored via different magnetic histories [10]. However, the periodic lattices favor stochastic switching of unit cells challenging the fidelity of reprogrammed excitations. This challenge has motivated researchers to address further lattices such as artificial spin ice (ASI) [11,12] in which geometrical frustration tailors magnetic states and excitations [13-18]. Experimental studies on AMQs are in their infancy [4-7] though AMQs are particularly interesting: (i) after saturation an AMQ can contain magnetic configurations that serve as nucleation sites for nonstochastic reversal processes [19]; (ii) due to its manyfold rotational symmetries and aperiodicity an AMQ exhibits a magnon density of states that densely fills reciprocal space [20]. In this paper, we present a comprehensive experimental study and simulations on spin excitations in three two-dimensional planar quasicrystals, namely Penrose P2, P3, and Ammann tilings of different rotational symmetry [Figs. 1(a)-1(c) and Table I]. We study them in saturation and the hysteretic regime by applying an in-plane magnetic field $\mathbf{H}$ in differ-

*vinayak.bhat@epfl.ch

†dirk.grundler@epfl.ch ent spatial directions. Comparing experimental spectra on both quasicrystals and a reference periodic lattice [Fig. 1(d)] with micromagnetic simulations we identify and categorize characteristic SW modes. Motivated by the recently reported nonstochastic switching in AMQs [19] we quantify internal bias fields $H_{\mathrm{B}}$ for the different tilings. Our analysis and results open the pathway to reprogrammable magnonics with quasicrystals.

\section{EXPERIMENTAL DETAILS}

Large $\left(2.4 \times 2.4 \mathrm{~mm}^{2}\right)$ lattices of nanomagnets were patterned on Penrose P2 (P2T) [4], Penrose P3 (P3T) [21,22], Ammann (AAT) [5], and square (SQT) lattices (Fig. 1) using nanofabrication techniques (see Supplemental Material [23]). The length and thickness of a given $\mathrm{Ni}_{80} \mathrm{Fe}_{20}$ (Py) segment were $810 \mathrm{~nm}$ and $25 \mathrm{~nm}$, respectively; the nominal width of a given nanobar for P2T, P3T, AAT, and SQT was 130, 130, 100 , and $130 \mathrm{~nm}$, respectively. Assuming isolated nanobars and disregarding the vertices we estimated relevant demagnetization factors $N_{x}$ (along the long axis), $N_{y}$ (across the width), and $N_{z}$ (in out-of-plane direction) using Ref. [24] (see Supplemental Material [23]). Room-temperature broadband spin-wave spectroscopy was performed in a flip-chip configuration [14] (see Supplemental Material). Simulations were performed using the OOMMF code $[14,25]$ on finite-size quasicrystals (see Supplemental Material).

\section{RESULTS AND DISCUSSION}

Eigenfrequencies measured at a fixed field value $\mu_{0} H=$ $100 \mathrm{mT}$ for different angles $\phi$ are shown in Figs. 1(e)-1(h). The experimental data show prominent (main) branches for each of the four samples (black/white contrast). They exhibit ten-, ten-, eight-, and fourfold rotational symmetry, respectively, which deviates from the lattice symmetry in case of P2 and P3 (Table I). We attribute the observation of a tenfold 


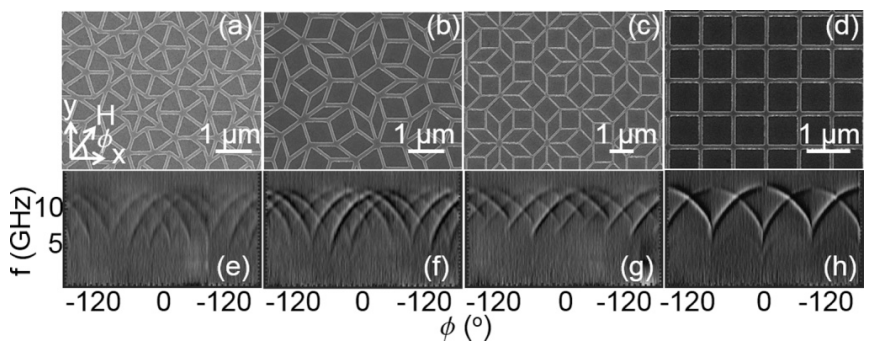

FIG. 1. Scanning electron microscopy images of inner sections of samples (a) P2T, (b) P3T, (c) AAT, and (d) SQT. Bright (dark) regions correspond to $\mathrm{Py}(\mathrm{GaAs})$. Grayscale plots summarizing spinwave absorption spectra measured at $\mu_{0} H=100 \mathrm{mT}$ as a function of angle $\phi$ for (e) P2T, (f) P3T, (g) AAT, and (h) SQT. To enhance the contrast we show difference spectra taken between neighboring data sets (derivative).

rotational symmetry to the linear polarization of microwave field in a coplanar waveguide (CPW). Hence containing, both left- and right-circularly polarized electromagnetic waves, the CPW excites the gyrotropic spin precession [26] for opposing field directions applied to the same segment of the AMQ. As a consequence, spin-wave spectra measured on a fivefold rotationally symmetric lattice show a tenfold rotational symmetry with respect to $\phi$. Branches are slightly hysteretic consistent with $H<H_{\text {ani }}$. Local frequency maxima in Figs. 1(e)-1(h) indicate that for the corresponding angle $\phi$ the magnetization vectors $\mathbf{M}$ of a subgroup of nanobars are parallel with both $\mathbf{H}$ and their easy axis. In this field orientation such nanobars exhibit the maximum internal field that enters the equation of motion and governs the precession frequency of spins [26]. Maximum frequencies are found to amount to about $12-13 \mathrm{GHz}$ at $100 \mathrm{mT}$ for all samples. Figure 2 shows spectra taken at fixed $\phi$ when $\mu_{0} H$ was decreased from $+100 \mathrm{mT}$ in a stepwise manner. For each lattice we depict spectra when field $\mathbf{H}$ was applied in two different directions.

In Figs. 2(a)-2(d) a pronounced single mode is seen in each lattice (local minima highlighted by upward triangle) when $\mu_{0} H$ is reduced to a small negative field of $-20 \mathrm{mT}$ (bottommost spectra). The resonance frequency and amplitude of this mode decrease if $H$ is further diminished (from bottom to top). At a certain negative field value, a resonance (re)appears in each data set (downward triangle) whose signal strength and frequency steadily increase with more negative $H$. The emergence of such a high-frequency mode indicates that nanobars, which were initially aligned against the negative field direction have reversed their magnetization. At a field of $-65 \mathrm{mT}$ the high-frequency mode reaches a saturated signal strength, which might indicate that irreversible switching processes were completed. When we analyzed spectra

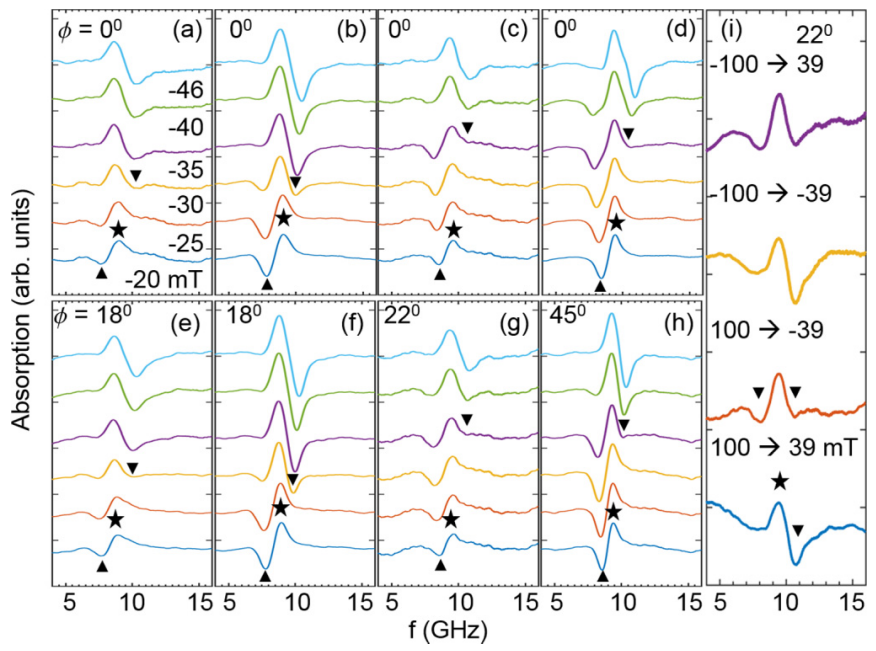

FIG. 2. Absorption spectra measured for different $\mathbf{H}$ at $\phi=0^{\circ}$ on (a) P2T, (b) P3T, (c) AAT, and (d) SQT. From each spectrum we subtracted the reference spectrum at $\mu_{0} H=0 \mathrm{mT}$. The asterisks indicate the positive resonance peaks (maxima) belonging to the reference spectrum in all graphs. Spectra taken on (e) P2T at $\phi=$ $18^{\circ}$, (f) P3T at $\phi=18^{\circ}$, (g) AAT at $\phi=22^{\circ}$, and (h) SQT at $\phi=45^{\circ}$. Field values (labels) are in $\mathrm{mT}$ and allocated to differently colored spectra in (a), and are valid until (h). The solid upward (and downward) triangles highlight resonances belonging to the main branches A before (and after the beginning of) the reversal. Notice the (re)appearance of the main mode with decreasing $H$ indicated by the downward triangles. (i) SW spectra measured on AAT in four different states at $\mu_{0}|H|=39 \mathrm{mT}$ and $\phi=22^{\circ}$ after saturation at large $|H|$ : (1) nearly saturated states in opposing fields [first (cyan) and third (yellow) curve from the bottom], and (2) partially reversed AAT (hysteretic regime) at opposing fields suggesting disordered states [second (red) and fourth (purple) curve from the bottom]. For (2), the two SW spectra are similar and different from (1), indicative of reprogrammable magnonics. Labels indicate field values (in $\mathrm{mT}$ ) for the relevant magnetic history.

obtained at the same field value $\mu_{0} H$ in successive field sweeps from $+100 \mathrm{mT}$ to $-100 \mathrm{mT}$ and back we observed reproducible resonance features also when $H$ resided in the regime of irreversible processes [Fig. 2(i) and Fig. S2 in the Supplemental Material]. Reproducible spectra in partially reversed magnetic configurations are a prerequisite for reprogrammable magnonics. We attribute the reproducibility to nonstochastic switching [19] of identical nanobars enabled by a self-biasing effect in the quasicrystalline lattices due to different local environments. For photonic quasicrystals it was stated that an identical single defect produced different localized states depending on its specific placement and local dielectric environment [27]. In the following we will argue

TABLE I. Bias fields $H_{\mathrm{B}}$ evaluated from branches A using Eq. (1) compared to demagnetization field values calculated for isolated nanobars (last column).

\begin{tabular}{lcccrr}
\hline \hline Lattice & Lattice Symmetry & Magnetic Symmetry & Main Branch & \multicolumn{1}{c}{$\mu_{0} H_{\mathrm{B}}(\mathrm{mT})$} & $\mu_{0} N_{x} M_{\mathrm{S}}(\mathrm{mT})$ \\
\hline Penrose P2 & fivefold & tenfold & $A_{P 20}$ & $2.98 \pm 0.35$ & 24.4 \\
Penrose P3 & fivefold & tenfold & $A_{P 30}$ & $8.2 \pm 0.37$ & 24.4 \\
Ammann & eightfold & eightfold & $A_{A T 0}$ & $8.39 \pm 0.38$ & 22.4 \\
Square & fourfold & fourfold & $A_{S 0}$ & $22.7 \pm 0.19$ & 24.4 \\
\hline \hline
\end{tabular}




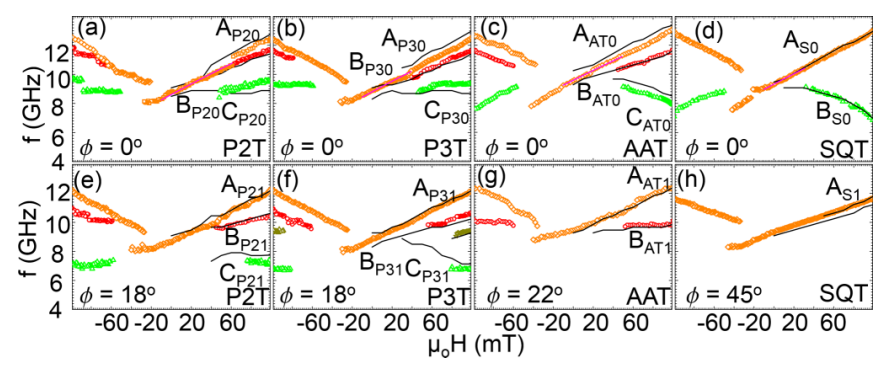

FIG. 3. Experimental resonance frequencies (symbols) obtained for different applied field, $H$, values for (a) P2T at $\phi=0^{\circ}$, (b) P3T at $\phi=0^{\circ}$, (c) AAT at $\phi=0^{\circ}$, (d) SQT at $\phi=0^{\circ}$, (e) P2T at $\phi=18^{\circ}$, (f) P3T at $\phi=18^{\circ}$, (g) AAT at $\phi=22^{\circ}$, and (h) SQT at $\phi=45^{\circ}$. The applied field was varied from $+100 \mathrm{mT}$ to $-100 \mathrm{mT}$. The solid black lines represent the simulated resonance frequency values. Magenta lines demonstrate Kittel formula fits to the branches representing SW modes at $\phi=0^{\circ}$.

that spin-wave resonance frequencies of the constituents of AMQs monitor different magnetic environments in analogy to the defect in a photonic quasicrystal.

In Fig. 3 we present the field-dependent resonance frequencies (symbols), which we extracted from large sets of spectra taken under conditions identical to Fig. 2. Besides the main mode, we identified further resonances (branches) of small signal strength. We encode branches in three different colors with labels $\mathrm{A}, \mathrm{B}$, and $\mathrm{C}$.

For P2T shown in Figs. 3(a) and 3(e), we see three branches at $+100 \mathrm{mT}$. In Fig. 3(a) for $\phi=0^{\circ}$, we label them $A_{P 20}$, $B_{P 20}$, and $C_{P 20}$. The branches $A_{P 20}$ and $B_{P 20}$ merge with each other for decreasing $\mu_{0} H$ at around $36 \mathrm{mT}$. The branch $C_{P 20}$ exists down to $29 \mathrm{mT}$. At $H=0$ only one branch is resolved, which dies out for negative fields. At about $-23 \mathrm{mT}$ (onset field), branch A reappears. This high-frequency branch is attributed to magnets with $\mathbf{M}$ parallel to both $\mathbf{H}$ and their easy axis direction. Branches $\mathrm{B}$ and $\mathrm{C}$ (re)emerge at $-65 \mathrm{mT}$ and $-54 \mathrm{mT}$, respectively. We attribute the different onset fields to reversal fields of subgroups of nanobars that exhibit specific misalignment angles with $\mathbf{H}$. All onset field values are smaller compared to $\mu_{0} H_{\text {ani }}$ indicating that reversal does not take place via coherent rotation [28]. The observation of the three branches at $\mu_{0} H<-65 \mathrm{mT}$ is taken as an indication that the nanobars forming P2T have reversed to a large extent.

In Fig. 3(e) for $\phi=18^{\circ}$, we label the three distinct branches by $A_{P 21}, B_{P 21}$, and $C_{P 21}$. The frequency separation between branches $A_{P 21}$ and $B_{P 21}$ is larger compared to $A_{P 20}$ and $B_{P 30}$ at $100 \mathrm{mT}$. Hence nanobars exhibit a larger variation in internal fields for this angle $\phi$. Branches $A_{P 21}$ and $B_{P 21}$ merge into a single branch at about $40 \mathrm{mT}$. This branch further decreases with decreasing $\mu_{0} H$. At $-27 \mathrm{mT},-58 \mathrm{mT}$, and $-60 \mathrm{mT}$ three branches successively appear that show a mirrored behavior compared to $A_{P 21}, B_{P 21}$, and $C_{P 21}$ at large positive $H$. Several branches are extracted for P3T [Figs. 3(b) and 3(f)] and AAT [Figs. 3(c) and 3(g)] as well. There exist, however, characteristic discrepancies: branches A to $\mathrm{C}$ change their slopes $d f / d H$ from lattice to lattice. At the same time the onset field values are different. Comparing eigenfrequencies at around $+40 \mathrm{mT}$ branches $\mathrm{A}$ and $\mathrm{B}$ are found to split more and more in frequency from lattice to lattice (left to right in Fig. 3).

In Figs. 3(d) and 3(h) we show the results obtained for the periodic square lattice SQT. For $\phi=45^{\circ}$ in Fig. 3(h) only a single branch is resolved at $100 \mathrm{mT}$. Here all the nanobars experience the same misalignment with respect to the applied field $\mathbf{H}\left(45^{\circ}\right)$. Correspondingly, the internal fields and eigenfrequencies are the same for all nanobars. In Fig. 3(d) at $\phi=$ $0^{\circ}$ two branches A and B of opposing slopes are identified for SQT. The positive (negative) slope $d f / d H$ at $H>0$ is attributed to nanobars being collinear (orthogonal) to $\mathbf{H}$ [26].

In the following we present an analysis of spectra in that we make use of the demagnetization factors and model the most prominent branches in terms of uniform precession in a nanobar. By this means, we discuss reasons behind different slopes $d f / d H$ of branches A focusing on $\phi=0^{\circ}$. We assume that the local environment of nominally identical nanobars induces a (self-)bias magnetic field $H_{\mathrm{B}}$. To estimate this field, we consider the Kittel equation for an individual magnetic ellipsoid in which we introduce $H_{\mathrm{B}}$ as an additional magnetic field [26]

$$
f=\frac{\gamma}{2 \pi} \sqrt{\left[\mu_{0}\left(H+H_{\mathrm{B}}\right)+\left(N_{z}-N_{x}\right) \mu_{0} M\right]\left[\mu_{0}\left(H+H_{\mathrm{B}}\right)+\left(N_{y}-N_{x}\right) \mu_{0} M\right]} .
$$

Here, $H_{\mathrm{B}}=0$ would represent an isolated nanobar without interacting neighbors. Its internal field is ruled by the demagnetization effect; its eigenfrequency is described by the unmodified Kittel equation. $H_{\mathrm{B}}=N_{x} M_{s}$ indicates that neighboring magnets compensate for the demagnetization field along a nanobar's long axis (i.e., they cancel magnetic charges) and induce a quasistatic internal field of zero similar to an infinitely long stripe. We fit Eq. (1) to branches A in Figs. 3(a)-3(d) (magenta lines behind the blue symbols for $H>0$ ). Using Eq. (1) we quantitatively model the branches. Table I summarizes the bias magnetic field $\mu_{0} H_{\mathrm{B}}$ evaluated for nanobars inside the four different lattices. The limiting case of $H_{\mathrm{B}}=N_{x} M_{s}$ is nearly fulfilled for SQT $\left(\mu_{0} H_{\mathrm{B}}=\right.$ $22.7 \mathrm{mT}$ ) where nanobars collinear with $H$ form stripelike chains. A considerably smaller value for the self-biasing field $\mu_{0} H_{\mathrm{B}} \cong 3 \mathrm{mT}$ is extracted in case of P2T. Here, the local environment only weakly compensates for the demagnetization field. The eigenfrequency comes close to uniform precession of an isolated nanobar. Values $H_{\mathrm{B}}$ are larger for P3T and AAT compared to P2T. Hence, quasicrystals of the same rotational symmetry such as P2T and P3T exhibit clearly different bias fields. Interestingly, P3T with a smaller nanobar density compared to P2T exhibits the larger $\mu_{0} H_{\mathrm{B}}$ of $8.2 \mathrm{mT}$. The eightfold symmetric Ammann lattice, which appears again denser than P3T shows an only slightly larger value (Table I). The design of the lattice is decisive for $H_{\mathrm{B}}$ acting on a subgroup of nanobars contributing to a specific branch. We speculate that the lattice-inherent magnetostatic 

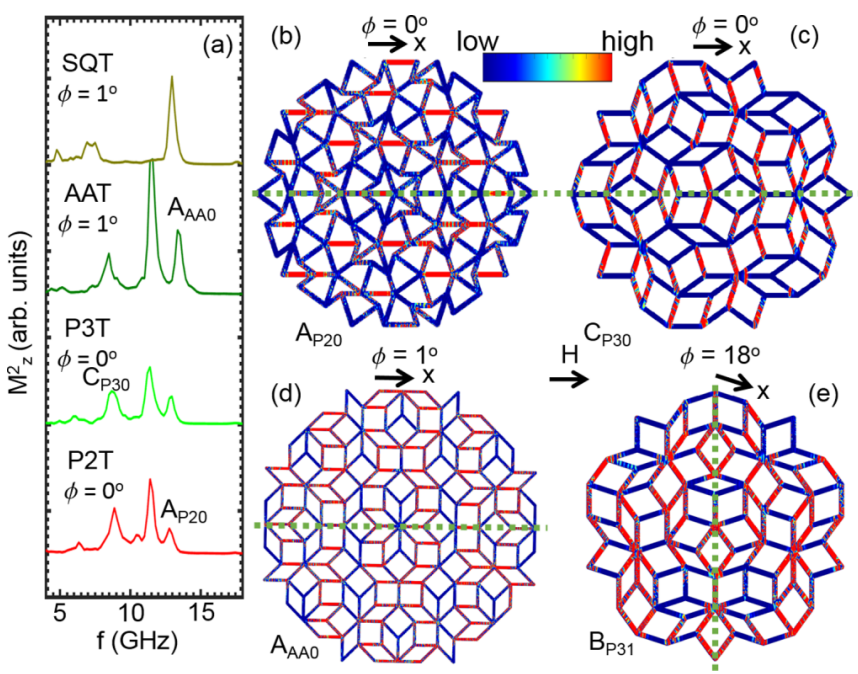

FIG. 4. (a) Simulated power spectra as a function of frequency for different lattices and in-plane angles at $\mu_{0} H=100 \mathrm{mT}$. The labels above each curve indicate the respective lattice and the inplane angle, $\phi$. Simulated local power map for $H=100 \mathrm{mT}$ for (b) P2T at $f=12.79 \mathrm{GHz}, \phi=0^{\circ}$, (c) P3T at $f=8.69 \mathrm{GHz}, \phi=0^{\circ}$, (d) AAT at $f=13.38 \mathrm{GHz}, \phi=1^{\circ}$, and (e) P3T at $f=10.25 \mathrm{GHz}$, $\phi=18^{\circ}$. The green dotted lines represent mirror axes. Red (blue) color corresponds to $2 \mathrm{k}(\mathrm{A} / \mathrm{m})^{2}\left[0 \mathrm{k}(\mathrm{A} / \mathrm{m})^{2}\right]$ values in square of spin precession amplitudes $\left(\mathrm{M}_{z}\right)$, respectively.

field $H_{\mathrm{B}}$ is important to understand not only different eigenfrequencies of the nominally identical nanobars but also nonstochastic switching reported for AMQs [19]. $H_{\mathrm{B}}$ leads to an inhomogeneous energy landscape and locally modifies the probability for reversal in an opposing field. The methodology and evaluation developed here help to quantitatively explore this phenomenon in different AMQs and functionalize them for reprogrammable magnonics. Here reproducibly accessible spectra in partially reversed AMQs are required [compare the spectra shown in Fig. 2(i) and in the Supplemental Material].

To understand in detail the microscopic origin of SW mode branches found in Figs. 2 and 3 in the saturated regime, we performed OOMMF simulations [25] in which we fixed the applied field along horizontal axis and rotated the bitmap geometry by angle $\phi$ with respect to the applied field direction. Spectra simulated for $\mu_{0} H=100 \mathrm{mT}$ are depicted in Fig. 4(a) for the four different samples. Here peaks indicate resonance frequencies. Simulations performed at many different field strengths and field orientations [see Fig. S3(a) for power spectra at different in-plane angles] provided us with resonance frequencies that we summarize as black lines in Fig. 3. We find a good agreement for the number of branches between experiment and simulation.

Considering the consistency we show the square of spatial distributions of spin-precessional amplitudes in Figs. 4(b)4(d), which we attribute to the modes of branches A at $\phi=0^{\circ}$. Here, the magnetization of nanobars collinear with the $x$ direction (field direction) is found to precess pronouncedly (red). Their precession is largely uniform supporting the modeling based on the Kittel formula [Eq. (1)]. We clearly observe that the modes in Figs. 4(b)-4(d) are mirror symmetric with respect to axes shown with green dotted lines. The axes are parallel to H. Surprisingly, when the field is applied along an off-symmetry axis $\left(\phi=18^{\circ}\right.$ for P3T), we again observe the existence of a mirror axis [Fig. 4(e)]. However, now the axis is perpendicular to $\mathbf{H}$. Further power maps on the quasicrystals suggest that branches $\mathrm{B}$ and $\mathrm{C}$ at large $\mu_{0} H$ can be interpreted as follows: for P2T, $B_{P 20}$, and $C_{P 20}$ arise from nanobars with angles $\pm 36^{\circ}$, and $\pm 72^{\circ}$, respectively, with respect to $\mathbf{H}$ consistent with the observed tenfold rotational symmetry. $B_{P 21}$ of $\mathrm{P} 2 \mathrm{~T}$ correlates to spin precession in nanobars oriented at $\pm 54^{\circ}$. Similar allocations hold true for P3T. For AAT and $\phi=0^{\circ}$, branches $A_{A A 0}, B_{A A 0}$, and $C_{A A 0}$ seem to belong to nanobars at $\phi=0^{\circ}, \pm 45^{\circ}$, and $\pm 90^{\circ}$, respectively. For $\phi=22^{\circ}$ two $\mathrm{SW}$ modes $A_{A A 1}$ and $B_{A A 1}$ arise from nanobars making an angle of $\phi= \pm 22^{\circ}$, and $\pm 68^{\circ}$, respectively, with respect to $\mathbf{H}$. Overall prominent branches observed at the distinct angles $\phi$ considered in Figs. 2 and 3 reflect spin precession in subgroups of nominally identical nanobars, which due to the long-range order are oriented in mirror symmetry under specific angles relative to $\mathbf{H}$. Their exact eigenfrequency is governed by the local environment of the subgroups. In Fig. 4(d) the prominently excited nanobars form bandlike patterns extending in a direction perpendicular to $\mathbf{H}$ applied along $x$ direction. These bands of coherently excited nanobars do not exhibit translation symmetry along $x$ direction due to the underlying quasicrystalline lattice. Simulations shown in Fig. S4 of the Supplemental Material indicate that mode symmetries and patterns are robust against edge defects. Further studies are required to understand how mode profiles vary when magnetic disorder and topological defects [29] are present. We note that in the regime of irreversible switching we found reproducible spectra for accordingly disordered quasicrystals (Fig. S2 in the Supplemental Material). Our results presented here provide the basis for studies addressing quasicrystals as exotic artificial spin ice structures incorporating topological defects [30].

\section{CONCLUSIONS}

To summarize, we studied artificial quasicrystalline ferromagnets based on Penrose P2, P3, and Ammann tilings. We observed systematic variations and reproducible series of field-dependent resonance frequencies across the hysteresis loops. The detailed comparison between experiment and simulations in the saturated regime indicates that the shape anisotropy of the individual nanobars played a dominant role for the value of the resonance frequency when considering the bias-field effect of the different long-range-ordered local environments. Penrose P2 and P3 tilings are found to exhibit striking similarities concerning SW modes residing in nanobars with orientations that are mirrored with respect to the applied field. Resonances in the Ammann tiling could be explained along a similar line assuming correspondingly different angles. Our experiments and findings pave the way for studies on reprogrammable magnonics based on quasicrystals and the spin dynamics of topological defects in exotic artificial spin ice.

\section{ACKNOWLEDGMENTS}

The research was supported by the Swiss National Science Foundation via Grant No. 163016 and Nanosystems Initiative Munich II funded by the Deutsche Forschungsgemeinschaft. 
V.S.B. thanks B. Farmer and I. Stasinopoulos for their help in editing the PYTHON code for Penrose P3 tiling in Ref. [22] to generate dxf files, and for the technical help in fabrication of CPW, respectively.
[1] B. Charrier and D. Schmitt, J. Magn. Magn. Mater. 189, 165 (1998).

[2] Z. Islam, I. R. Fisher, J. Zarestky, P. C. Canfield, C. Stassis, and A. I. Goldman, Phys. Rev. B 57, R11047(R) (1998).

[3] C. Nisoli, R. Moessner, and P. Schiffer, Rev. Mod. Phys. 85, 1473 (2013).

[4] V. S. Bhat, J. Sklenar, B. Farmer, J. Woods, J. T. Hastings, S. J. Lee, J. B. Ketterson, and L. E. De Long, Phys. Rev. Lett. 111, 077201 (2013).

[5] V. S. Bhat, J. Sklenar, B. Farmer, J. Woods, J. Ketterson, J. T. Hastings, and L. E. De Long, J. Appl. Phys. 115, 17C502 (2014).

[6] V. Brajuskovic, F. Barrows, C. Phatak, and A. Petford-Long, Sci. Rep. 6, 34384 (2016).

[7] D. Shi, Z. Budrikis, A. Stein, S. A. Morley, P. D. Olmsted, G. Burnell, and C. H. Marrows, Nature Phys. 14, 309 (2018).

[8] S. Nikitov, P. Tailhades, and C. Tsai, J. Magn. Magn. Mater. 236, 320 (2001).

[9] A. V. Chumak, A. A. Serga, and B. Hillebrands, J. Phys. D 50, 244001 (2017)

[10] M. Krawczyk and D. Grundler, J. Phys.: Condens. Matter 26, 123202 (2014).

[11] R. Wang, C. Nisoli, R. Freitas, J. Li, W. McConville, B. Cooley, M. Lund, N. Samarth, C. Leighton, V. Crespi et al., Nature (London) 439, 303 (2006).

[12] Y. Qi, T. Brintlinger, and J. Cumings, Phys. Rev. B 77, 094418 (2008).

[13] L. Heyderman and R. Stamps, J. Phys.: Condens. Matter 25, 363201 (2013).

[14] V. S. Bhat, F. Heimbach, I. Stasinopoulos, and D. Grundler, Phys. Rev. B 93, 140401(R) (2016).

[15] V. S. Bhat, F. Heimbach, I. Stasinopoulos, and D. Grundler, Phys. Rev. B 96, 014426 (2017).

[16] M. B. Jungfleisch, W. Zhang, E. Iacocca, J. Sklenar, J. Ding, W. Jiang, S. Zhang, J. Pearson, V. Novosad, J. Ketterson et al., Phys. Rev. B 93, 100401(R) (2016).
[17] X. Zhou, G.-L. Chua, N. Singh, and A. O. Adeyeye, Adv. Funct. Mater. 26, 1437 (2016).

[18] Y. Li, G. Gubbiotti, F. Casoli, F. Gonçalves, S. Morley, M. Rosamond, E. Linfield, C. Marrows, S. McVitie, and R. Stamps, J. Phys. D 50, 015003 (2016).

[19] V. Bhat, B. Farmer, N. Smith, E. Teipel, J. Woods, J. Sklenar, J. Ketterson, J. Hastings, and L. De Long, Physica C 503, 170 (2014).

[20] J. Rychly, S. Mieszczak, and J. Klos, J. Magn. Magn. Mater. 450, 18 (2017).

[21] M. Gardner, Penrose Tiles to Trapdoor Ciphers: And the Return of Dr Matrix (Cambridge University Press, Cambridge, 1997).

[22] P. Jeff, http://preshing.com/20110831/penrose-tilingexplained/.

[23] See Supplemental Material at http://link.aps.org/supplemental/ 10.1103/PhysRevB.98.174408 for experimental spin wave spectra for different angles and magnetic fields in switching and hysteretic regimes for P2, P3, AAT, and SQT. Furthermore, simulated spin wave spectra for different tilings can also be seen.

[24] A. Aharoni, J. Appl. Phys. 83, 3432 (1998).

[25] M. Donahue and D. Porter, Interagency Report NISTIR 6376, 1999.

[26] A. G. Gurevich and G. A. Melkov, Magnetization oscillations and waves (CRC Press, Boca Raton, 1996).

[27] Z. V. Vardeny, A. Nahata, and A. Agrawal, Nature Photon. 7, 177 (2013)

[28] J. M. D. Coey, Magnetism and Magnetic Materials, 4th ed. (Cambridge University Press, Cambridge, 2014).

[29] S. Gliga, A. Kákay, R. Hertel, and O. G. Heinonen, Phys. Rev. Lett. 110, 117205 (2013).

[30] B. Farmer, V. S. Bhat, A. Balk, E. Teipel, N. Smith, J. Unguris, D. J. Keavney, J. T. Hastings, and L. E. De Long, Phys. Rev. B 93, 134428 (2016). 